\title{
Patrones para la gestión de la información en las páginas World Wide Web de las organizaciones
}

\author{
Marina Vianello Osti \\ Departamento de Biblioteconomía y Documentación \\ Universidad Carlos III de Madrid
}

\subsection{Resumen}

Reflexión acerca de los modelos adoptados por las organizaciones para la gestión de las páginas Web. Se observa en que medida estos modelos inciden en la calidad de la información y se analizan tres tipos de estructura de sitios Web: centralizado, descentralizado y cooperativo. Se enfatiza en la necesidad de adoptar unos modelos basados en la cooperación y en la gestión descentralizada de los flujos de información, que basen su funcionamiento en la función mediadora del documentalista/bibliotecario.

Palabras Claves: World Wide Web. Hipertexto. Internet. Intranet. Gestión de la información.

\subsection{Abstract}

Reflections on the models adopted by the organizations for WWW-based information systems management. The influence of such models on the quality of the information is examined, analizing three Web structures: centralized, decentralized and cooperative. The necessity of adopting models based on cooperation and decentralized management of the flows of information is emphazised, and relying on the intermediating role of the Documentalist/Librarian is advised.

Keywords: World Wide Web. Hypertext. Internet. Intranet. Informtion management.

\section{Introducción}

Las tecnologías de la información representan una herramienta imprescindible que facilita enormemente la producción de documentos y reduce los tiempos de acceso a los mismos. El fenómeno Internet, y sobre todo, la aparición de la 
World Wide Web nos proporcionan la posibilidad de acceder a una cantidad de información inimaginable hasta hace poco tiempo.

Se podría pensar que todo esto favorecerá un gran desarrollo individual e impulsará enormemente el progreso científico. Sin embargo, las enormes potencialidades de las tecnologías de la información pueden no materializarse, si junto a ellas no se dan los cambios que llevan implícitos en la organización del conocimiento, en los procesos de tomas de decisiones y en la distribución del poder dentro de las organizaciones.

En los últimos años la gran popularidad alcanzada por la World Wide Web ha impulsado, y casi impuesto, a numerosas organizaciones a mantener una presencia en la red y proporcionar información a través de este medio a un público cada vez más numeroso. No obstante, nos encontramos con que a menudo muchos de los sistemas informativos frustran las expectativas de sus usuarios debido a una deficiente arquitectura del sistema, producto de una gestión de la información mal planificada, o debido a que no nos ofrecen aquello que necesitamos.

Se pretende hacer una reflexión acerca de los modelos que las organizaciones han adoptado para la gestión de la información, y queremos observar en qué medida inciden en la calidad de los productos. El enfoque que adoptamos en nuestro análisis deriva del convencimiento de que el simple transporte de información no representa transmisión de conocimientos, sino que éstos se producen a través de una organización mental impulsada por una intención. La información se transforma en conocimiento a través de un flujo complejo de interacciones. Desgraciadamente, resulta demasiado frecuente que el diseño de los sistemas de información se aborde privilegiando los aspectos tecnológicos, desde el convencimiento de que el diseño de las metodología informática pueda por sí sola ser garantía de éxito y olvidando la dimensión social del problema, que hace necesaria para su resolución una convergencia pluridisciplinar. Desde esta óptica hay que resaltar que los bibliotecarios/documentalistas se deberán enfrentar con nuevos retos para el aprovechamiento de las oportunidades que este panorama les proporciona. Deben ser conscientes de que su presencia representa un factor clave para la gestión de la información en las empresas y, más específicamente, en el ámbito hipertextual.

\section{Los sistemas informativos de las organizaciones}

Los Management Information Systems (MIS), nacen al inicio de los años 60 y son la expresión de la necesidad de las empresas de contar en su interior con unos eficientes flujos informativos que faciliten la toma de decisiones. Esta necesidad ha supuesto desde entonces el reto de conjugar las tecnologías de la infor-

Scire. 5 : 2 (jul.-dic. 1999) 23-45. 
mación con las problemáticas de la organización empresarial. El objetivo perseguido siempre ha sido la creación de unos sistemas de información unitarios, a menudo centrados en exceso en las tecnologías de la información, que han olvidado considerar los factores sociales del trabajo en grupo, la organización de los sistemas de producción y la plasmación de los conceptos en un contexto informativo automatizado. Al mismo tiempo los MIS han centrado su objetivo en producir, almacenar y dirigir la información, sin plantearse la selección de la misma para evitar información inútil y sobrecargas; en definitiva, sin preguntarse cuál es el patrimonio informativo empresarial realmente significativo (Gaio y Mich, 1997).

El fracaso de estas experiencias ha producido frecuentemente el descontrol informativo dentro de las organizaciones, de modo que las diversas áreas, para satisfacer sus necesidades específicas, han ido creando y organizando sus propios recursos con la proliferación de servicios periféricos. Pocas son las empresas españolas que están en condiciones de contestar afirmativamente a las preguntas de si disponen de la información que necesitan; si la que poseen está distribuida adecuadamente; y si se utiliza de forma oportuna en la toma de decisiones (Martín Megias, 1998, p. 553). Según este mismo autor "la función y responsabilidad de la gestión de la información en la empresa están dispersas en distintos departamentos, sin que ninguno de ellos asuma la misión de aprovechar adecuadamente las sinergias que se producen entre todas y cada una de las funciones afectadas" (Ibidem, p.554).

A la situación antes descrita, en los últimos años se ha sumado el extraordinario desarrollo de la World Wide Web, que ha puesto en evidencia la posibilidad de utilizar sus técnicas para la gestión de la información dentro de las organizaciones, dando lugar a la creación de las Intranet para la gestión integrada de todo tipo de datos internos a través del navegador Web, que permite acceder de forma hipertextual a las varias funciones de la organización en el mismo momento en que se producen. Al mismo tiempo se ha hecho cada vez más apremiante para las organizaciones la necesidad de estar presentes en la red para un público cada día más numeroso. Todo esto no ha hecho sino aumentar la necesidad de soluciones para la gestión de la información, puesto que no es suficiente resolver los problemas internos de la empresa, cuyos miembros son a la vez productores y usuarios de la información, sino que hay que planificar también un servicio orientado a los usuarios externos, garantizándoles una información rica, fiable y con todas las herramientas necesarias para que su uso sea lo más fácil posible.

Las informaciones que las organizaciones suelen proporcionar a través de sus servicios Web son:

Scire. 5 : 2 (jul.-dic. 1999) 23-45. 
- Información general acerca de la organización, dirigida a los usuarios externos que pueden querer conocer la organización o ponerse en contacto con ella.

- Información estrictamente relacionada con la función de la organización en relación con sus usuarios externos (ej.: los cursos de una entidad académica).

- Instrumentos e información auxiliares a la actividad de la organización.

Lo lógico, por lo tanto, es postular que Intranet y servicio Web mantengan una relación de ósmosis cuyo filtro entre el interior y el exterior consista en los oportunos cortafuegos, y que la información generada por la propia organización se refleje hacia el exterior proporcionando una imagen dinámica y actual, abierta a la sociedad.

\section{El World Wide Web}

El World Wide Web, a diferencia de otros sistemas hipermedia tradicionales, permite acceder a un gran variedad de servicios de forma dinámica e interactiva, gracias a un interfaz independiente tanto de la ubicación física de la información como de la aplicación que la ha creado, y que al mismo tiempo permite al gran público el acceso a la información de manera intuitiva, proporcionando a las organizaciones una visibilidad cuya ventajas no pueden ser descuidadas.

La Web está concebida de tal forma que permite a los autores estructurar la información a través de una red asociativa de documentos conectados entre sí por unos enlaces que los liberan de organizar los contenidos de forma lineal, como sucede con los documentos impresos, al mismo tiempo el lector del documento hipertextual adquiere un papel mucho más activo, teniendo la posibilidad de elegir el camino y construyéndose su propio recorrido de lectura en función de sus intereses.

\subsection{Estructura del hipertexto}

El nodo es la unidad mínima de información en un hipertexto. Dentro de un nodo la información se presenta organizada en módulos y debe ser claramente delimitada. Teóricamente debería tratar de una sola "idea", que se puede relacionar con otra a través de unos enlaces. Los nodos de información pueden ser de diverso tipo : definiciones, atributos, referencias, notas, ilustraciones, ejemplos.... Estos nodos normalmente se visualizan a través de un título, un icono. Las ideas están en los nodos. Un conjunto de nodos es una red que puede corresponder a la estructura de un tema o a una red semántica creada por el usuario.

Los enlaces son el elemento estructural que permite fundar la coherencia en el sentido de continuidad textual en el hipertexto. Ponen en relación los nodos o 
bloques informativos y permiten al usuario ejercer la lectura. Es el conjunto de los enlaces lo que estructura el documento y funda la relación semántica de acercamiento de contextos dentro del mismo hipertexto y, como en el caso de la World Wide Web, de circunstancias remotas.

Pascual Cantos Gómez, Francisco Javier Martínez y Gregorio Moya (1994) distinguen dos categorías atendiendo al destino del enlace y al propósito del mismo.

- Según el destino de la relación:

- Externos e internos, según se produzca el enlace entre nodos pertenecientes al mismo documento o a documentos distintos.

- De conexión entre aplicaciones, enlaces que una vez activados cargan un programa que crea unos efectos colaterales en la navegación (por ejemplo, lanza una búsqueda a un motor o robot de recuperación) y que pueden modificar los recorridos de la lectura.

— De comando, aquéllos que diseñan el interfaz de lectura y controlan la navegación.

- Según el propósito de la relación:

— Estructurales y semánticos: los primeros organizan estructuralmente el documento y son jerárquicos, y los segundos permiten conectar nodos temáticos.

- Implícitos y explícitos

- Unidireccionales y bidireccionales

- Planos y definidos; los planos son aquéllos que se activan sin conocer el destino.

Enlazando los nodos a través de los enlaces se produce lo que se define como navegación: "se entiende por navegación un tipo de consulta especial donde el usuario accede a la información con entera libertad, saltando de un segmento de la misma a otro con total independencia y sin limitaciones del sistema" (Cantos; Martínez y Moya, 1994, p.33).

El lector podrá moverse exclusivamente dentro de un documento hipertextual específico siguiendo los enlaces estructurales, utilizando así el texto de la forma más secuencial posible y eliminando la dimensión hipertextual.

$\mathrm{Si}$ el lector utiliza los enlaces referenciales y procede por asociaciones, creará su propio camino.

Con este procedimiento, el lector acabará abandonando el hipertexto desde donde había empezado la navegación y se moverá enlazando documentos remotos. El progresivo alejamiento del punto de partida a veces no se produce de for- 
ma consciente y da lugar a la pérdida de la orientación, por lo cual el lector ya no sabe dónde se encuentra y ha perdido la noción de lo que estaba buscando.

Blunstein, Webber y Tague-Sutcliffe (1997) proponen una serie de principios en la organización de los enlaces para optimizar su uso y reducir la sensación de desorientación:

- Principio de no saturación: consiste en evitar la incertidumbre en la toma de decisión restringiendo el numero de los enlaces.

- Principio de explicitación: permite al usuario la identificación del tipo de relación que el enlace introduce.

- Principio de objetivación: supone el empleo de mecanismos objetivados de elección de los enlaces para evitar la dispersión que distintos analistas pueden provocar.

- Principio de adecuación: su planteamiento se debe realizar en conformidad con el resto de los elementos del sistema.

- Principio de efectividad: se debe procurar que las cadenas de enlaces para encontrar un documento no sean demasiado largas.

- Principio de ordenación: la disposición de los enlaces ha de estar ordenada.

- Principio de oportunidad: se ha de tender a cubrir todas las posibilidades de creación de enlaces sin contradecir el principio de no saturación (1).

\subsection{Los seis principios del hipertexto}

P. Lévy (1990, p.29-31) define el hipertexto a través de seis principios que son:

- Metamorfosis: La red hipertextual está en continua construcción y negociación.

Una publicación puede ser continuamente puesta al día, pueden pasar por ella numerosos lectores que le atribuirán un significado distinto dependiendo del contexto en que esté insertada. El mismo conocimiento humano comparte este principio, ya que no se puede considerar inmutable, sino que nuevas experiencias pueden cambiar de forma radical el significado que atribuimos a los objetos.

- Heterogeneidad: Los nodos y los enlaces de una red hipertextual son heterogéneos. El hipertexto puede unir con relaciones distintas objetos diferentes. También en nuestro cerebro los objetos del conocimiento son el producto de la unión de imágenes o sensaciones procedentes de diferentes dominios sensoriales. 
- Multiplicidad: El hipertexto se organiza según un modelo fractual, según el cual cada nodo o enlace puede ser reticular. Un hipertexto puede ser considerado el nodo de otro hipertexto. Puede resultar difícil distinguir dónde termina un hipertexto conectado en red. También nuestra actividad cognitiva funciona de manera análoga, una palabra en la mente activa una red de palabras, conceptos, imágenes, etc.

- Exterioridad: La red no posee una unidad orgánica ni un motor interno. No es posible saber con antelación cómo se desarrollará la red. Los desarrollos de un hipertexto son provocados por la interacción con fenómenos externos que no se pueden determinar internamente.

- Topología: En los hipertextos todo funciona según el principio de proximidad, de cercanía. La experiencia hipertextual tiene aspectos próximos a la exploración, la lectura hipertextual se aproxima a la experiencia de la interpretación de un mapa.

- Movilidad de los centros: La red no tiene centro, más bien posee muchos perpetuamente en movimiento. También la actividad neurológica, según Minsky, se organiza a través de procesos polícentricos.

El hipertexto, donde la información es representada por la acción que la genera, parece por tanto el instrumento más idóneo para ser aplicado a entornos complejos y en continua evolución, puesto que proporciona una herramienta flexible para adquirir, crear, explotar, acumular nuevos conocimientos. Al mismo tiempo su empleo pone de manifiesto la necesidad de formular nuevos criterios para su organización.

\section{Las informaciones de las organizaciones}

Las informaciones presentes en el sistema informativo de una organización deben reflejar la realidad que vive la institución en el momento en que se produce la consulta. Un cambio en la realidad de la organización deberá provocar un flujo informativo que modifique el contenido del sistema y, en determinados casos, también su arquitectura.

Rens Scheepers y Jan Damsgaard (1997) adoptaron, para el estudio de las Intranet, la teoría de la estructura social institucionalizada de A. Giddens (2), según la cual, acción y estructura se definen como una dualidad que proporciona una conexión entre la naturaleza humana y la estructura social. Los cambios que se producen en las organizaciones deben ser interpretados como alteraciones debidas a la acción, donde la nueva estructura que se genera es el medio y el resultado de la acción que ella organiza.

Al contrario de la interpretación tradicional que entiende por sistema el reflejo de una situación estable, y que por lo tanto centraliza y delimita las partes que

Scire. 5 : 2 (jul.-dic. 1999) 23-45. 
lo componen, es importante configurar sistemas que interpreten las alteraciones que en ellos se producen como fuentes enriquecedoras, abandonando el enfoque tradicional que separa los datos de los procesos que los generan.

Giddens aísla tres dimensiones en la estructura social institucionalizada: significación, legitimación y dominación; e identifica tres procesos clave para la acción humana que interactúan con estas tres dimensiones: comunicación, ejercicio del poder y sanciones de la conducta. Las dimensiones de la estructura se unen a los procesos de tres formas: esquemas interpretativos, medios y normas.

También nosotros emplearemos las tres dimensiones de Giddens para explicar la dinámica de un sistema de información de una organización.

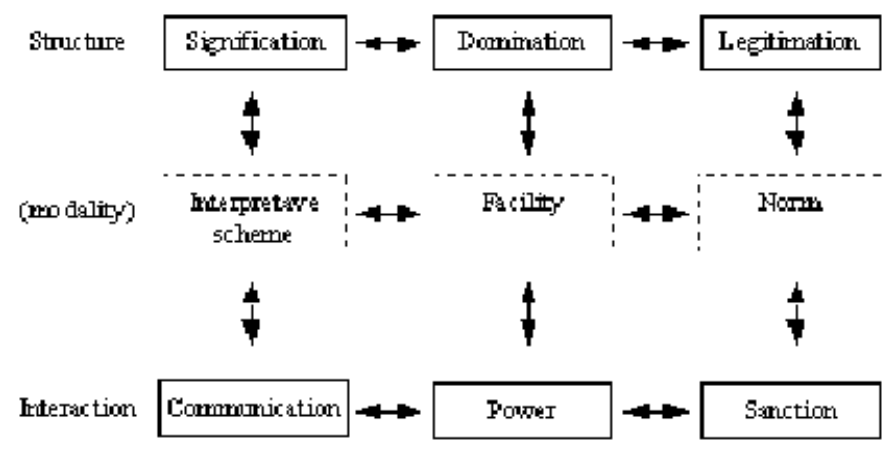

Fig.1. Fuente: Scheepers, 1997.

\subsection{Dimensión de significación}

La significación en el contexto de un sistema de información refleja la comprensión, compartida por un grupo, de la función de dicho sistema de información.

Los usuarios internos deberán compartir los mismos objetivos tanto para la creación como para el mantenimiento de la información, y tanto ellos como los usuarios externos deberán comprender su organización para poder sacar de ella el máximo provecho.

Sin embargo, gran parte de los problemas que se producen son debidos al desconocimiento por parte de los usuarios internos de su propio papel en la presentación de la organización hacia el exterior.

Frecuentemente las deficiencias detectadas en estos servicios (Gómez del Pulgar Rodríguez de Segovia, 1998; Vianello, 1999) suelen ser provocadas por el 
descuido de algunas de las normas estilísticas básicas para la confección de páginas Web (3).

Entre las carencias que podemos recordar, las más frecuentes son:

- Ausencia tanto de las fechas de creación como de las fechas de actualización de la información presentada, con grave perjuicio para la fiabilidad de la información suministrada.

- Ausencia de un teléfono de contacto, de la dirección y a veces incluso de un buzón de sugerencias, en detrimento de la participación y el intercambio informativo entre usuario interno y externo.

- Ausencia, en algunos casos, de instrucciones de navegación y de facilidades para volver desde algunas secciones informativas a la página principal.

- Ausencia de caracteres de identificación del organismo.

\subsection{Dimensión de dominio}

El dominio refleja las competencias atribuidas a los actores para actuar en el sistema. La fiabilidad de las informaciones generadas se determinará por el nivel de responsabilidad que reviste su origen, pudiéndolas dividir en actos formales, actos personales y fuentes alternativas que producen informaciones no documentadas formalmente, que sin embargo son importantes elementos de integración informativa.

Las informaciones oficiales (actas, reglamentos, etc.) son la clase de informaciones generadas por actos formales y que documentarán los principales cambios de la realidad institucional, necesitando un control mínimo y siendo generadas por los órganos representativos de la misma organización.

Las informaciones personales son aquéllas cuya responsabilidad recae sobre un individuo o grupo, que pueden ser consideradas oficiales por un grupo o una parte de la organización sin serlo para la organización en su conjunto, y que representan la gestión cotidiana.

Las informaciones recogidas son especialmente relevantes en la World Wide Web, a la cual se accede a través de enlaces hipertextuales, y permiten la incorporación al sitio de documentos que provienen de fuentes alternativas a la organización. Se trata de informaciones cuya producción suele ser ajena a la organización y en cuanto tal presentan particulares problemas de mantenimiento.

Las informaciones, además, son susceptibles de otra clasificación que centre su atención en la vigencia de los contenidos o, como algunos lo llaman, su nivel de volatilidad. Esta irá variando a lo largo de su vida en la organización. Tendremos informaciones actuales, que representan el estado actual de la organización; informaciones obsoletas, que ya no la representan; e informaciones his-

Scire. 5 : 2 (jul.-dic. 1999) 23-45. 
tóricas que, si bien ya no representan la realidad de la organización y han quedado obsoletas, pueden tener algún tipo de interés, por lo que en lugar de ser eliminadas se conservarán identificándolas con claridad.

\subsection{Dimensión de legitimación}

La estructura de legitimación reflejará las normas que definen los códigos de conducta dictados por la organización y que garantizan el uso apropiado de las aplicaciones. Define los procedimientos necesarios para mantener actualizado y coherente el sistema informativo, confiriendo las competencias para modificar y poner al día la información, responsabilidad que suele recaer en el administrador del sistema informativo, quien en algunos casos puede delegar sus derechos en otras estructuras. Del administrador o de sus delegados dependerá la validación, que consiste en el paso de una información a través de un control, para modificarla o aprobarla, y ponerla definitivamente a disposición del usuario externo.

Estas tres dimensiones deben ser interpretadas desde una visión circular, como aparece en la fig.1, donde toda alteración respecto al modelo provoca que el mismo vaya modificándose.

\section{Modelos de organización}

La puesta en marcha de un sistema de información supone definir un modelo de organización que refleje la dinámica de la organización, permitiendo una gestión coherente del servicio en función de las necesidades informativas, de los instrumentos de soporte y sus perspectivas de crecimiento.

Deberá garantizar que la estructura de significación sea coherente, vigilando que la presentación formal de los documentos respete unas consignas estilísticas entre cuyas finalidades está evidenciar la "imagen corporativa" de la organización y evitar las ambigüedades.

A partir del análisis de la estructura del dominio se definirá cómo se produce la interacción entre el sistema de información, los sistemas productores de la información y el resto de la organización. Se creará un mapa de los mecanismos de producción documental, se definirán las distintas clases de información y su flujo dentro de la organización y se designarán las responsabilidades tanto para el diseño como para el mantenimiento del sistema. Finalmente se organizarán de forma consecuente las estructuras de almacenamiento de la información, distribuidas en soportes heterogéneos.

Se definirá la política de legitimación, dejando sentados los criterios de validación según de qué información se trate, así como las responsabilidades para las modificaciones y las actualizaciones. Esto se reflejará en la organización de la clasificación de la información para su recuperación y difusión. 
Entre los modelos de organización de la información más frecuentemente adoptados por parte de los servicios World Wide Web existentes se han señalado, por un lado, el centralizado y el descentralizado (Axelsson,1995; Bragadini,1997) y, por otro el cooperativo (Bragadini, 1997).

\subsection{El modelo centralizado}

En los modelos centralizados se suele partir de la consideración según la cual la información es un recurso común de la organización que necesita ser controlado de manera similar a los otros tipos de recursos. Tendrá que estar disponible para todos en la empresa y presentar un carácter estable, lo cual será posible poniendo en común todos los datos, globalizando la información y separándola de las unidades que la han producido. A tal fin las organizaciones nombran un gerente de los recursos informativos.

La gestión de la información es por lo tanto llevada a cabo desde una única titularidad. El administrador del sistema y el responsable de su arquitectura son la misma persona, que decide cuál es la información que debe ser introducida y define qué datos estarán a disposición de los usuarios internos y cuáles de los usuarios externos. El mecanismo de comunicación va desde el usuario interno (productor de la información) al gestor del sistema, que recoge la información producida en las varias secciones de la organización y del cual siempre depende la validación final. A veces es el mismo gestor quien solicita las puestas al día de la información, en cuyo caso asume además las funciones de coordinador.

Este modelo fuertemente estructurado resulta válido sobre todo para la gestión de la información que, concluido su ciclo de creación, ha sido ya validada y considerada lo suficientemente estable para ser almacenada en una bases de datos. Es importante a este propósito subrayar que "sólo el $20 \%$ de la información con la que trabaja una empresa está almacenada de forma estructurada [en] bases de datos relacionales" (Eito, 1998, p.7).

Las ventajas de este modelo son evidentes sobre todo para los usuarios externos, ya que este tipo de organización fuertemente estructurada proporciona datos coherente y una presentación de la información homogénea, factores ambos que protegen contra la desorientación.

Por el contrario, este modelo responde mal a los cambios que se pueden producir en la estructura de la organización y que pueden hacer necesarias modificaciones en su arquitectura. Suele tardar en reflejar tales modificaciones y a menudo la imagen se alejará de la realidad de la organización.

Su defecto de más inmediata percepción suele ser la deficiente puesta al día de los datos, ya que las unidades productoras de la información, al no tener auto- 
nomía para organizar su propia información, pueden no sentirse implicadas en el proyecto, no entender su alcance y no colaborar o hacerlo mal.

Al mismo tiempo, su incapacidad para reflejar la actividad del día a día no le permite trasmitir la dinámica de la organización, pudiendo provocar que el personal de la organización, desmotivado por su escaso apego a los acontecimientos, deje de usarlo y recurra a otros sistemas de información alternativos.

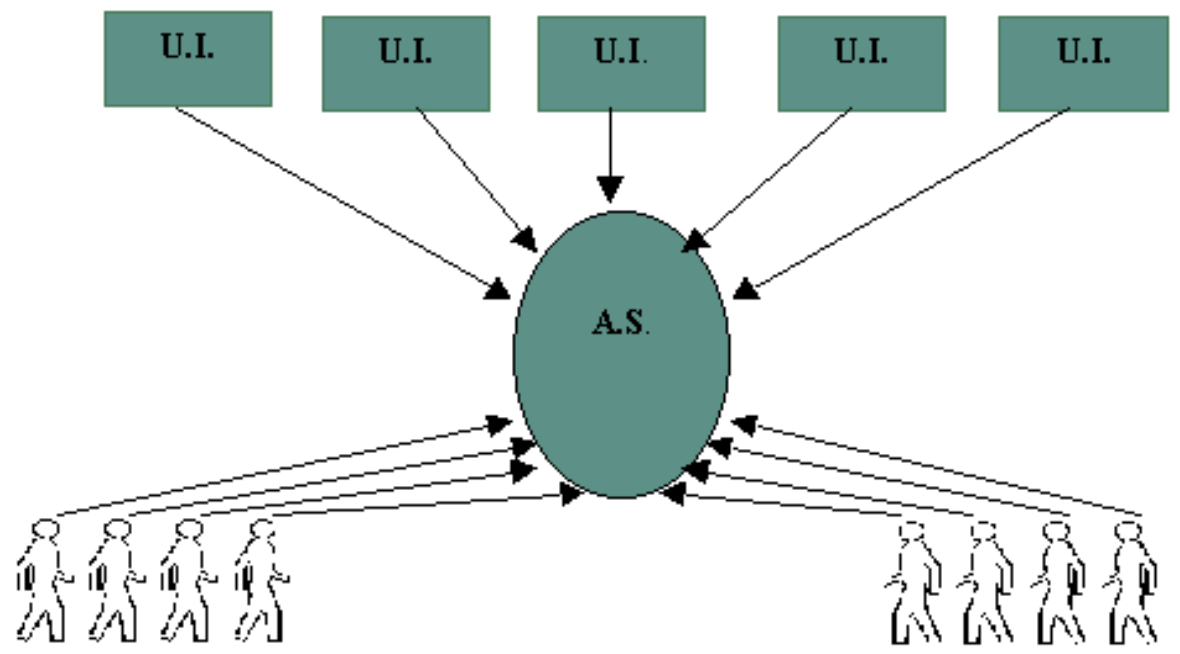

Usuarios intermos

Usuarios externos

Fig. 2. Modelo centralizado de organización de la información

La figura 2 muestra cómo las Unidades de Información (U.I) de la organización deben suministrar la documentación al Administrador del Sistema, que tiene toda la responsabilidad para su definición, organización y validación. Los usuarios, tanto externos como internos, accederán a la información una vez que el administrador la haya organizado.

\subsection{EI modelo descentralizado}

El modelo descentralizado, se sitúa en el polo opuesto respecto al modelo centralizado, y se suele producir cuando el desarrollo de los servicios informativos World Wide Web se ha ido creando desde servicios periféricos, a través de la acumulación de servicios sustancialmente independientes. La información se 
difunde y organiza donde se ha producido, cada servicio es el responsable de sus sistemas de información, con un responsable técnico o administrador, que asume a pequeña escala las competencias del responsable del sistema. Puede no existir una figura que asuma el control global de la arquitectura del sistema o puede haber una unidad que se haga cargo de la información de la organización en sus aspectos generales y comunes a todas las unidades que la componen.

En este tipo de estructura la validación y puesta al día de la información corresponde a cada una de las unidades y suele ser más eficaz, dada la proximidad del responsable a los artífices de la información (que además a menudo suelen ser alguno de ellos). Sin embargo, los mecanismos de comunicación de los cambios o actualizaciones de la información a la unidad que mantiene la información general (si existe), se producen exclusivamente de forma voluntaria.

El resultado suele ser el frecuente solapamiento de las temáticas que son comunes a más de una unidad, mientras al mismo tiempo se pueden quedar otras áreas sin documentar. La causa de este "descontrol" en muchos casos se puede atribuir al hecho de que en una primera etapa la presencia en la red de las organizaciones se ha producido por iniciativa de departamentos técnicos, que no siempre han podido reflejar de manera completa la organización, y también al hecho de que a este diseño inicial se hayan ido integrando otros servicios sin pasar por un nuevo proyecto o una verdadera planificación.

Las desventajas de cara al usuario externo son evidentes, ya que este modelo resulta difícil de utilizar. No ofrece suficiente garantía acerca de la oficialidad de la información, pues a veces faltan claras indicaciones acerca de la responsabilidad de la información. Resulta muy difícil en este tipo de estructura proporcionar al usuario externo un mapa del sitio y esto, junto con las incongruencias propias de sitios que han ido creciendo sin un plan, acentúa la sensación de desorientación, pérdida de tiempo e información.

De cara a los usuarios internos, sin embargo, este tipo de organización resulta quizás más motivadora, ya que su participación, mucho más directa, en la creación del servicio los implica más. Esto repercute en una mayor vitalidad informativa y en una puesta al día de los documentos mucho más ágil. A pesar del riesgo de duplicaciones, este tipo de modelo suele proporcionar mayor información, desde el momento en que la atención no está centrada solamente en ofrecer un escaparate hacia el exterior, sino que se alimenta por intereses personales.

La figura 3 muestra la relación entre diferentes Unidades de Información. Cada U.I posee un Administrador que está en condiciones de seleccionar, organizar, modificar y validar la información. Al carecer de un elemento de coordinación, se pueden producir solapamientos en la información. Los usuarios

Scire. 5 : 2 (jul.-dic. 1999) 23-45. 


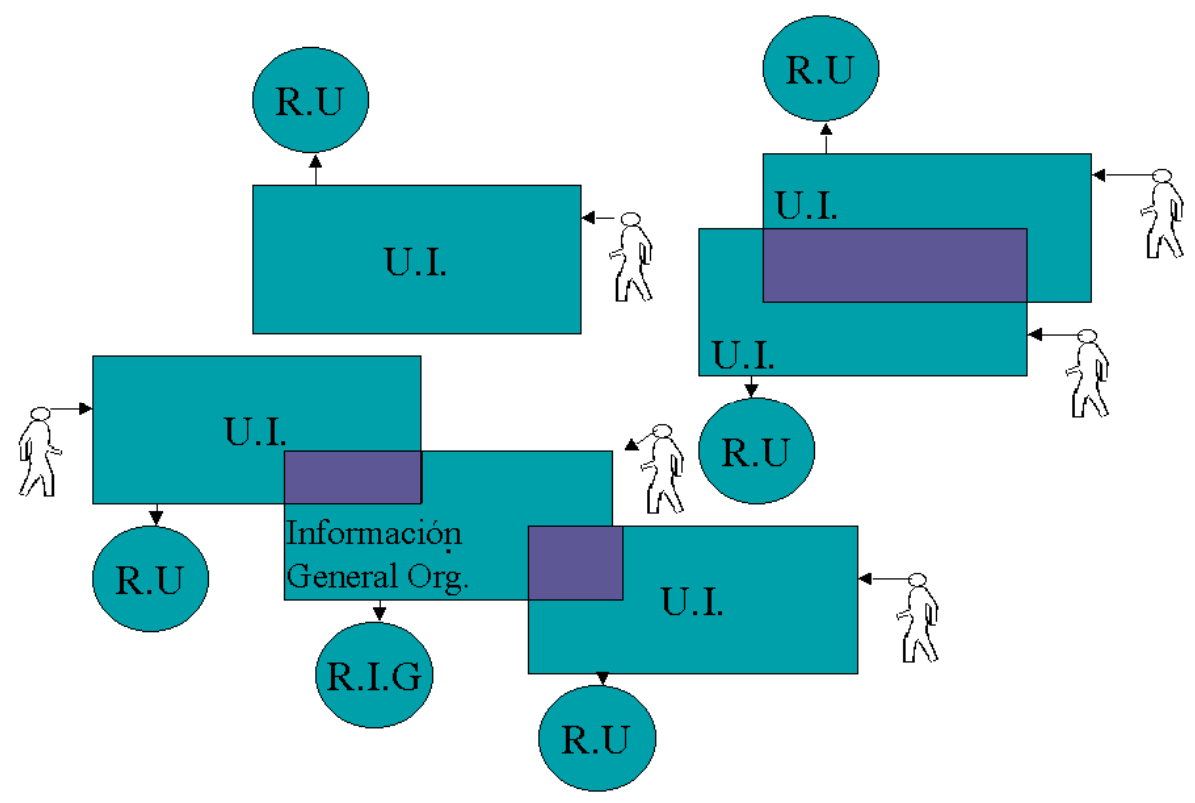

Fig. 3. Modelo descentralizado de organización de la información

podrán acceder a la información directamente a través de los servicios que la han originado.

\subsection{El modelo cooperativo}

Un intento de superación de las deficiencias de los dos modelos anteriores viene representado por el modelo cooperativo, diseñado para permitir la participación en su funcionamiento a las varias unidades de la organización sin que por eso se produzca una acumulación indiscriminada de la información. La responsabilidad de su creación recae en una única persona, cuyas competencias son diseñar la arquitectura del sistema asegurando su coherencia global, basada tanto en el conocimiento del entorno de la organización como en los objetivos individuales y colectivos. Es responsable además de definir unos criterios normalizadores para la presentación y la confección de la información, de velar para que se respeten, garantizar la fiabilidad de los datos y definir los criterios de recuperación de la información.

Este modelo cooperativo se estructura alrededor de dos ejes: la formalización de unas reglas que definan los limites y ámbitos de responsabilidad, y el estudio del ciclo de vida del documento. 
Con el estudio del organigrama de la organización se identifican las unidades implicadas en la creación documental, a las cuales, según su tipología, se atribuye el nivel de responsabilidad que le corresponde en cada una de las fases del ciclo de vida de los documentos (mecanismos de comunicación/distribución/destrucción).

Bragadini (1997) propone y analiza algunos modelos basados en esta arquitectura y plantea la conveniencia de que el técnico del sistema y el administrador de la arquitectura informativa sean figuras separadas, el primero encargado del soporte técnico del sistema y el segundo responsable de identificar las unidades informativas de la organización y el diseño de los mecanismos de comunicación en su interior.

La formalización del modelo se basa en la localización de las unidades informativas y en la definición de las clases de información producidas. Las unidades informativas deben coincidir con las unidades productoras de información, siempre que sea posible adquieren directamente la responsabilidad sobre la información que producen y el responsable de la arquitectura nombra un "gestor de la unidad".

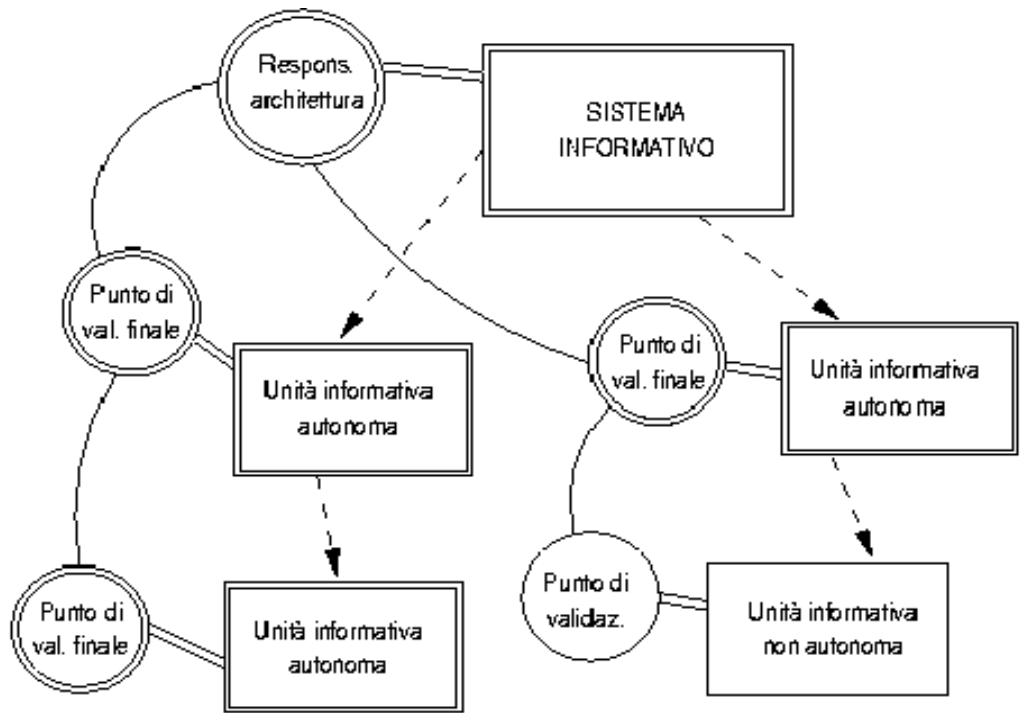

Fig. 4. Modelo de organización cooperativa (Fuente: Bragadini, 1997)

Scire. 5 : 2 (jul.-dic. 1999) 23-45. 
El titular responsable de la unidad a su vez puede delegar en otros, éstos en otros y así sucesivamente. Esta cadena de delegaciones que el autor llama "árbol de las delegaciones" tiene como finalidad reducir al máximo los pasos necesarios para validar los documentos y agilizar así su puesta a disposición de los usuarios: “...los procedimientos de validación se efectúan solo una vez, en abstracto, sobre el titular y el dominio de la unidad en el momento de la delegación...”(Bragadini,1997).

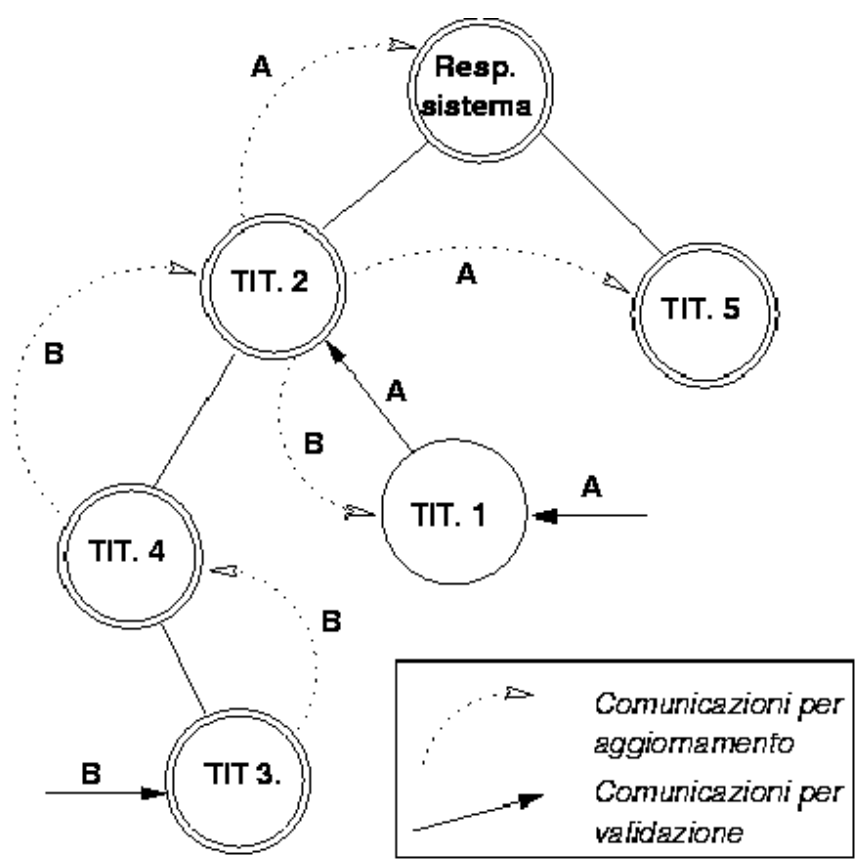

Fig. 5. Árbol de las delegaciones(Fuente: Bragadini, 1997)

La figura 5 muestra un diagráma que representa un árbol de las delegaciones. Es un ejemplo de los mecanismos de la comunicación: la modificación del dato A es validada por el punto 1, que no siendo el punto final de la cadena de las delegaciones, lo pasa al punto 2, que es el final. El titular 2 envía y distribuye el documento a todos los titulares de su mismo nivel y al responsable del sistema. La modificación de B es inmediatamente operativa en cuanto 3 es un punto final, 
luego vuelve hasta al titular 2, que no la difunde a todo el sistema sino sólo a nivel interno al titular 1.

El empleo de este modelo, a diferencia del modelo centralizado, contribuye a la difusión de las experiencias y los conocimientos técnicos, ya que a través del sistema de delegaciones implica en el mantenimiento del sistema a una amplia representación de la organización.

Sin embargo, el hecho de que la responsabilidad estratégica para la definición de las unidades y la atribución de las responsabilidades dependa, exclusivamente, del responsable de la arquitectura, desvirtúa en parte la carga cooperativa del modelo, que al fin y al cabo ve su nacimiento determinado por una estricta visión jerárquica. Identificando a las unidades productoras de información, y en su interior al responsable final del producto, se reproduce el modelo clásico de la gestión que fragmenta las organizaciones en departamentos, especializando las funciones y la tareas. Esto es factible en el sector de productos tangibles, pues se trabaja con procesos lineales, cuyas secuencias se conocen y se pueden establecer de antemano y, además, porque se sabe lo que se debe lograr al final del proceso. Sin embargo, esto es imposible cuando el producto es la información.

\section{Nuevas tecnologías e información cooperativa}

La llegada de las autopistas de la información introduce nuevas prácticas intelectuales y nuevas organizaciones del trabajo. Esto hace que los sistemas basados en los tradicionales modelos de gestión de la información no respondan a las nuevas exigencias de la sociedad e impulsa investigaciones orientadas a proporcionar soluciones que permitan las transferencias de los conocimientos, donde la dimensión tecnológica coexista con la atención a los mecanismos sociales y cognitivos.

La circulación de los documentos en soporte electrónico produce un flujo de información que continuamente pone en discusión los sistemas de representación que se han establecido. En este contexto, algunos investigadores han propuesto unos sistemas que, en oposición a las formulaciones cerradas de la inteligencia artificial, se fundan en los principios de la auto-organización. Estos principios tienen su origen en el estudio de las funciones cerebrales. Queremos recordar entre ellos los trabajos de Maturana y Varela (1987) que han abierto una nueva perspectiva de estudio de los sistemas cognitivos. Estudiando los fenómenos biológicos, han formulado la hipótesis de que el control global y la representación simbólica no existen. El comportamiento de un sistema es instituido a través de cooperaciones que se producen localmente. El comportamiento global de un sistema complejo no es fruto del control ejercitado por un dispositivo central, emerge de la organización de las conexiones internas y de las asociaciones con el

Scire. 5 : 2 (jul.-dic. 1999) 23-45. 
entorno. El cerebro humano posee una capacidad auto-organizadora, trabaja de forma distribuida y sus conexiones se transforman en el curso de la experiencia. La idea de una mente donde la información entra, se elabora y produce acciones u otras informaciones, da paso a la visión de una mente que se automodifica como consecuencia de sus interacciones con el ambiente (4).

Entre las aplicaciones emergentes de estas nuevas tendencias recordamos:

- El Workflow es un conjunto de dispositivos técnicos cuya aplicación se asocia a las funciones de programas clientes como los GED (Gestión Electrónica de los Documentos) y que permite definir, administrar, guiar y ejecutar los flujos de informaciones que se producen dentro de un grupo de trabajo.

El Workflow tiene su origen en la teoría de la coordinación y comunicación de Fernando Flores y Terry Winograd, quienes a partir de los años 80 desarrollan una nueva interpretación del papel del ordenador en las comunicaciones. Ellos sostienen que la mayoría de la coordinación humana se produce a través de acciones, peticiones y compromisos, y plantean que el ordenador, en lugar de ser usado como simple procesador de datos, pueda ser empleado para facilitar este tipo de coordinación. (Winograd; F.Flores, 1987).

El empleo de las aplicaciones Worlflow se debe basar en el análisis de los procedimientos llevados a cabo a través del llamado Business Process Reengineering (BPR), el cual proporciona una visión de los procesos que privilegia los aspectos intra-funcionales, plantea la necesidad de reestructurar ex novo los procesos y pone de manifiesto la importancia dentro de las organizaciones de los factores humanos, culturales y organizativos que deben acompañar los cambios tecnológicos (Gaio y Mich, 1997).

- Los sistemas basados en el tratamiento del lenguaje natural, el análisis matemático de los flujos documentales y el empleo de modelos de redes neuronales para generar la puesta al día dinámica de los mapas estratégicos. Recordamos, entre otros, el trabajo de W.Turner (1995), que ha diseñado un sistema cuya finalidad es analizar en qué medida el flujo de la información influye en nuestra percepción y determina nuestras decisiones (Fig. 6).

- Las aplicaciones basadas en las técnicas multi-agentes para la búsqueda de información en soporte electrónico y su adaptación a entornos dinámicos. Señalamos entre ellas el trabajo de Gleizes y Glize (1999). 


\section{El papel de los documentalistas}

En este nuevo contexto, autores como García Jiménez (1998, p.315) resaltan que las funciones del profesional de la información serán:

- Selección de la información, tanto de la que reside en el sistema como de aquélla cuyo acceso se produce a través de la red.

- Tratamiento y almacenamiento, manteniendo y organizando la documentación de las bases de datos, pero también proporcionando a los productores de los documentos orientaciones prácticas para la normalización de las presentaciones, uso de los enlaces, etc..

- Creación de productos documentales, creación y mantenimiento de los enlaces con las fuentes internas o externas.

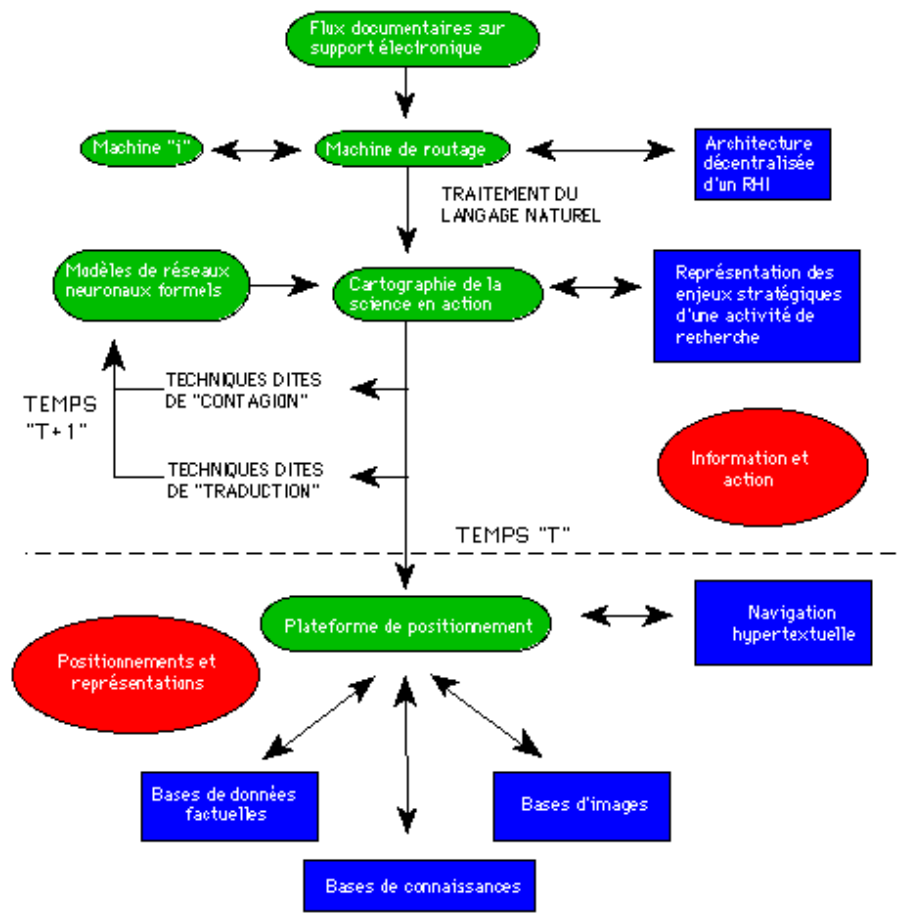

Objets d'étude

Mayens d'étude

Traitements informatiques

Fig. 6. Árbol de las delegaciones (Fuente: Turner. 1995)

Scire. 5 : 2 (jul.-dic. 1999) 23-45. 
- Recuperación de la información, evaluación permanente de las herramientas diseñadas a estos efectos y distribución selectiva de la información.

- Formación de los usuarios.

- Participación en el diseño y en el mantenimiento de los sistemas y su evaluación.

- Cooperación con los departamentos productores de información y participación con los directivos en el diseño de los flujos de trabajo.

Evidentemente el profesional de la información deberá asumir un papel en el que la aplicación de las técnicas ya tradicionales de la documentación deberá ser acompañada por competencias que el uso de las nuevas tecnologías hacen imprescindibles; sin embargo, pensamos que esto no se debe interpretar solamente como un incremento de competencias sino que debería reflejarse en un replanteamiento de la profesión.

Alfons Cornellá (1998a), reflexionando sobre un artículo de Rashi Glazer, profesor de la Universidad de Berkeley, en California (5), concluía que la información no tiene un valor per se, no se puede valorar como si fuesen bienes ya que, a diferencia de éstos, tiene valor económico sólo cuando es usada, y este valor se lo atribuye el usuario. La previsión de estos objetivos no es posible en un entorno dinámico y cualquier sistema que se base en definiciones construidas $a$ priori está destinado a perder rápidamente su eficacia y necesitará por tanto ser redefinido. El documentalista tendrá que ser sobre todo un mediador o, como escribe Cornellá (1998b), ocuparse de las transacciones de información.

Esta actividad mediadora creará las bases para la circulación de la información, ya sea dirigida al interior de la organización como al exterior; sin embargo, estos flujos informativos no se deberían modelar reproduciendo la estructura de producción documental, sino atendiendo a sus proyectos y objetivos.

\section{Conclusiones}

Las organizaciones necesitan como fuente de recursos la red y al mismo tiempo ya no pueden aplazar su presencia en la misma. Las Intranet y los servicios de información Web necesitan unos mecanismos que permitan que se alimenten el uno del otro para crear un flujo de información dinámico y enriquecedor. Desde esta perspectiva hemos observado los modelos de sistemas de información que más frecuentemente se han adoptado para satisfacer estas exigencias. Ninguno de ellos, en nuestra opinión, saca verdadero partido de las posibilidades que nos brindan Intranet e Internet. 
El modelo centralizado presenta una respuesta insuficiente frente a los cambios informativos de las organizaciones, y la separación que se produce entre el producto y el productor enfría los deseos participativos del personal.

El modelo descentralizado representa sin duda un modelo a descartar, ya que su desarrollo frecuentemente es fruto de una acumulación indiscriminada de la información.

El modelo cooperativo intenta superar los límites de los anteriores y consigue implicar al conjunto de la organización en su creación, al mismo tiempo que ofrece una visión global de la misma, gracias a la presencia de un responsable de su arquitectura. Sin embargo, se desarrolla según una concepción representacionista, basada en la atribución de delegaciones, de tipo descendente, o sea, jerárquica. Este sistema separa la información de la acción que la produce y fija el esquema de su funcionamiento a priori. El modelo sigue interpretando la gestión de la información interna/externa como si fuera un producto tangible cuya producción siguiera unos procesos lineales, conocidos y establecidos de antemano. Seguramente este modelo puede ayudar a mejorar la puesta al día de la información de las World Wide Web, sin embargo no soluciona la raíz del problema. Es necesario asumir una actitud de adaptación al cambio.

Será por lo tanto necesario adoptar unos modelos que se basen en la cooperación y en la gestión descentralizada de los flujos de información, que, en lugar de definir estructuras, se construyan alrededor de los objetivos y que sean lo suficientemente dinámicos como para ser redefinidos puntualmente según las necesidades que vayan surgiendo. La información no se describirá según su tipología sino según sus funciones y las responsabilidades deberán distribuirse en función de los proyectos.

La responsabilidad de la organización de este tipo de sistema no podrá residir exclusivamente en un diseñador sino que necesitará de un mediador, este se hará cargo de que la información se intercambie, será el elemento que hará las transacciones informativas. La competencias tradicionales de los bibliotecarios/documentalistas, integradas con el manejo de las nuevas tecnologías reinterpretadas en conformidad con las exigencias que hemos expuesto, abrirán seguramente horizontes prometedores para la profesión.

\section{Notas}

(1) Tomado de Antonio García Jiménez (1998, p.319-320)

(2) Rens Scheepers y Jan Damsgaard fundamentan su adopción de la teoría de Giddens en algunas de sus obras principales (Giddens, 1979, 1982 y 1984).

(3) Entre las más conocidas normas de estilo se puede consultar el Yale Manual Sytle.

Scire. 5 : 2 (jul.-dic. 1999) 23-45. 
(4) Véase a este propósito el dossier n5 (1998) de Solaris enteramente dedicado a este tema.

(5) El articulo al cual se refiere Cornellá es de Glazer (1998).

\section{Referencias}

Axelsson, K. (1995). Centralized or decentralized responsability for information systems? Research Report LiTH-IDA-R-95-29. Linköping: Departament of Computer and Information Science, Linköping University, septiembre 1995.

Blunstein, J; Webber, R.E.; Tague-Sutcliffe, J. (1997). Methods for evaluating the quality of hypertext links. // Information Precessing \& Management. 33 : 2 (1997) 133-144.

Bragadini, A. (1997). Un modello cooperativo per la gestione di un sistema informativo World Wide Web. Tesis presentada a la Facoltá di Scienza dell'Informazione de la Universitá di Pisa. 1997. URL: 〈http://zamboni.dsnet.it/ alessio/tesi/node1.html〉 . Fecha de consulta: 25.7.1998.

Cantos Gómez, P. ; Martínez Méndez, F. J. ; Moya Martínez, G. (1994). Hypertexto y documentación. Murcia : Universidad de Murcia, 1994. p. 32-34.

Cornellá, A. (1998a). Medir el conocimiento es medir al "conocedor" . // Extra-Net. Mensaje 386. URL: <http://www.extra-net/articulos/ en981202.htm>. Fecha de consulta: 14.12.1998.

Cornellá, A. (1998b). Tecnologías, empresas, inteligencia. // Extra-net. Mensaje 376 (29/10/98). URL: 〈http://www.extra-net/articulos/ en981029.htm> . Fecha de consulta: 14.12 .1998 .

Eito Brun, R.(1998). Sistemas GED e indexadores Intranet: ¿Alternativas excluyentes o tecnologías complementarias?. // El profesional de la información. 7 : 9 (1998) 5-9.

Gaio, L.; Mich, L. (1997). Informática e azienda fra miti e realtá: spunti di discussione. Dipartimento di Informatica e Studi Aziendali. Universitá delgi Studi di Trento. 1997. URL: <http://www.ecs.unitn.it/ lgaio/tif/tif7.html> . Fecha de consulta: 19.11.1998]

García Jiménez, A (1998) La gestión y el tratamiento de la información hipermedia en las instituciones: organización del conocimiento e información electrónica. // VI ${ }^{\mathrm{a}}$ Jornadas Españolas de Documentación: Los sistemas de información al servicio de la sociedad. Valencia, del 29 al 31 de octubre. Valencia: FESABID, 1998. 313-322.

Glazer, Rashi (1998). Measuring the Knower: Towards a theory of Knowledge Equity. // California Management Review . 40 : 3 (1998). p. 175.

Gleizes, M.P; Glize, P. (1998). L'adaptation des organisations par et pour l'information émergente. // Solaris.5,1999. URL: <http://www.info.unicaen.fr/bnum/jelec/ No5\5glize.html> . Fecha de consulta: 28.2.1999.

Gómez del Pulgar Rodríguez de Segovia, G. (1998). La administración española en Internet: Evaluación de sus Webs. // VI Jornadas Españolas de Documentación: Los sistemas de información al servicio de la sociedad. Valencia, del 29 al 31 de octubre. Valencia: FESABID, 1998. p. 377-390. 
Giddens, A. (1979). Central Problems in Social Theory: Action, Structure and Contradiction in Social Analysis. Londres: Macmillan, 1979.

Giddens, A. (1982). Profiles and Critiques in Social Theory. Londres: Macmillan, 1982.

Giddens, A. (1984). The Constitution of Society: Outline of the Theory of Structuration. Cambridge: Polity Press, 1984.

Landow, G. (1990). Hypertext and collaborative work: the example of Intermedia. // Galegher, J.; Kraut, R.E.; Edigo, C. (ed.). Intellectual teamwork. Hillsdale, NJ: Lawrence Erlbaum Associates, 1990. p. 407-428.

Lévy, P.(1990) Les technologies de l'intelligence. París: La Découverte, 1990.

Martín Megias, P. (1998). De la auditoría de Información a Intranet: Claves para la implantación de sistemas de gestión de información en las empresas. // VI J Jornadas Españolas de Documentación: Los sistemas de información al servicio de la sociedad. Valencia, del 29 al 31 de octubre. Valencia: FESABID, 1998. p. 553-556.

Maturana, H.; Varela, F. (1987). L'Albero Della Conoscenza. Milano: Garzanti,1987.

Scheepers, R.; Damsgaard, J. (1997). Using Internet technology within the organization: a structurational analysis of intranets. // XX Information systems research seminar in Scandinavia. 1997. URL: 〈http://iris.informatik.gu.se/conference/iris20/29.htm〉 . Fecha de consulta: 23.11.1998.

Solaris. Dossier. 5 (1998). URL: <http://www.info.unicaen.fr/bnum/jelec/Solaris/d05/ index.html>. Fecha de consulta: 28.2.1999.

Turner, W.A.(1995). Penser l'entrelacement de l'human et du tecnique: les réseaux hybrides d'intelligence. // Solaris. 1. 1995. URL: <http://www.info.unicaen.fr/jelec/ Solaris/d01/1turner.html> . Fecha de consulta: 15.12.1998.

Vianello Osti, M. (1999). Para un estudio de la información Web de las universidades españolas. // III Encuentro de ISKO-España: Organización del conocimiento en sistemas de información y documentación. Getafe, 19 al 21 de noviembre de 1997. Zaragoza: Universidad, 1999. p. 465-473.

Winograd, T.; Flores, F. (1987). Calcolatori e conoscenza. Milano: Mondadori, 1987.

Yale Manual Sytle. URL: <http://www.cocc.edu/pcasey/manual/sites/site_covers.html〉. Fecha de consulta: 25.2.1999.

Scire. 5 : 2 (jul.-dic. 1999) 23-45. 